تصميم بطاقة موائمة للسيطرة على سرعة محرك تيار مستمر

عبر الممر الصناعي القياسي للحاسبة

$$
\text { فراس أحمد الارزي }
$$

مدرس مساعد في قسم علوم

كلية علوم الحاسبات والرياضيات /
د. عبد الإله خضر محمود

مدرس في قسم الهندسة الكهربائية

الحاسبات

كلية الهندسة / جامعة الموصل

جامعة الموصل

\title{
الخلاصة
}

تم في هذا البحث تصميم دائرة موائمة Data Acquisition Card كبطاقة ربطت عبر القبر

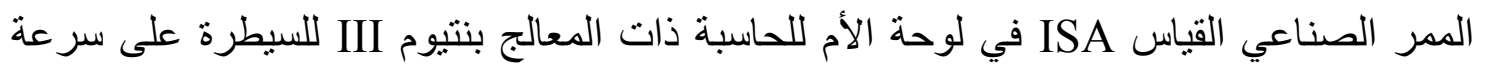

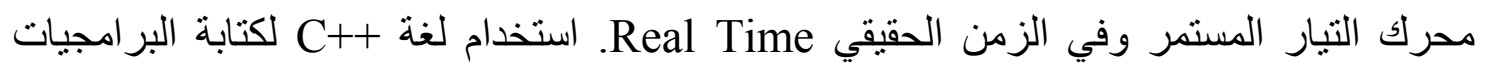

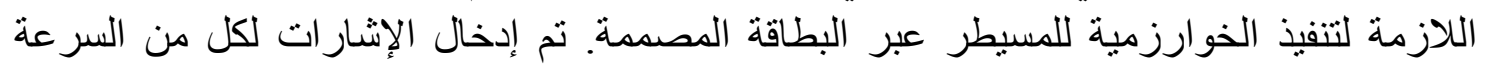

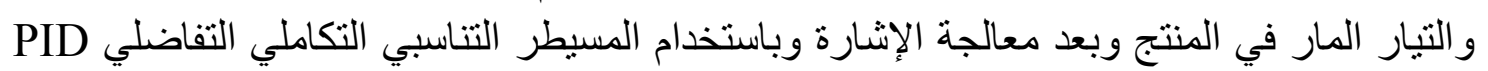

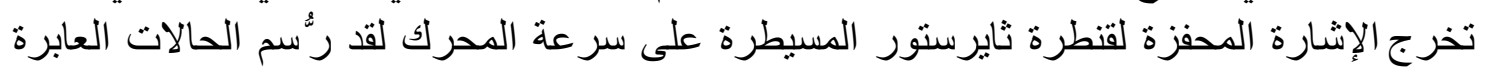

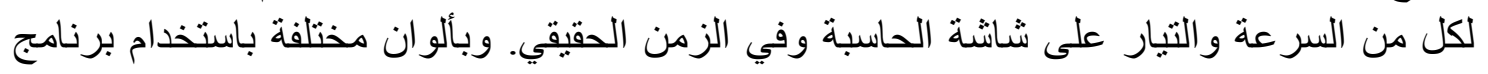

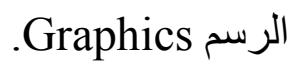

الكلمات الاليلة : بطاقة مو ائمة ، الممر القباسي الصناعي ISA ، السيطرة على سرعة محرك التيار المستمر ، مسيطر تناسبي تكاملي رقمي.

\section{Design of DAQ Card to Control the Speed of DC Motor Using ISA Slot}

Dr. Abdelelah K. M.

Firas Ahmed Alderzey 
محمود : تصميم بطاقة موائمة للسيطرة على سرعة محرك تيار مستمر عبر الممر الصناعي القياسي للحاسبة

College of Computer Science and Mathematics College of Engineering

Dept. of Computer Science

Dept. of Elect. Eng.

University of Mosul

University of Mosul

\begin{abstract}
Data acquisition card has been designed as a card connected to the industrial standard architecture (ISA) slot in the motherboard of a Pentium III personal computer in order to control the speed of a DC motor in real time. A program in $\mathrm{C}++$ language has been written to realize and implement an algorithm of the controller and to get in and out of the signals to and from the designed card. The two sampled signals, the speed of the DC motor and the current in the armature, are processed by using digital proportional plus integral (PI) control and the output signal is the actuating signal for a thyristor bridge to control the speed of DC motor. The transient response for current and speed are drawn in PC monitor in the real time with different colors using graphics.
\end{abstract}

Keywords: Data acquisition, card (DAQ), ISA, speed control, DC motor, PI controller.

قبل في 2006/9/11

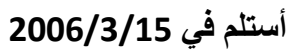

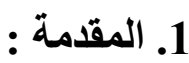

تنو عت البحوث المنشورة حول استخدام الحاسبة للسبطرة على سرعة محرك التبار المستمر

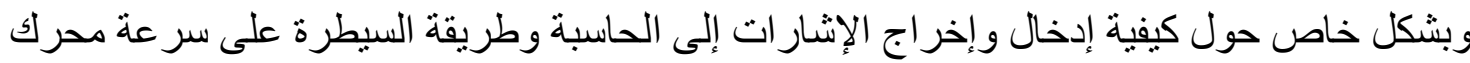

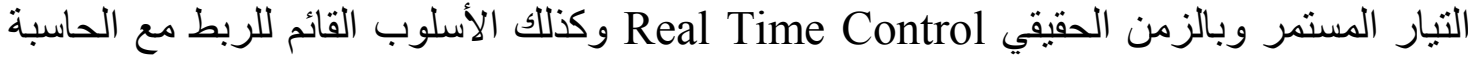


وتعثيق البيانات وإخر اج الإثارة المسيطرة إما بالأسلوب المتو الي أو المتوازي مع تطور الحاسبات

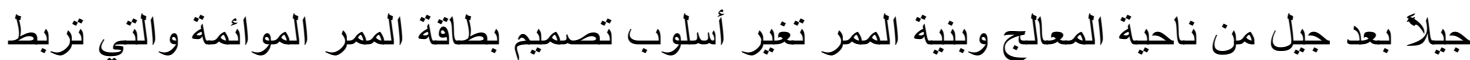

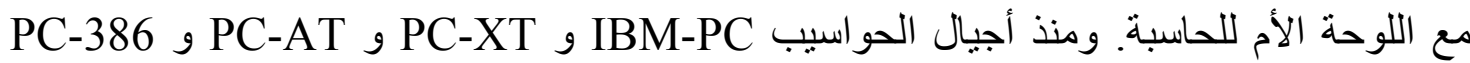

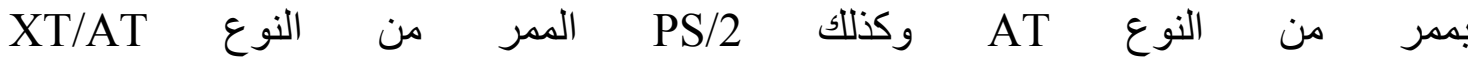
و PS/2 بمر القناة المايكروية وأنظمة الممر EISA و أنظمة الممر PC1 [1]].

والبحوث المنشورة في هذا المجال متتوعة وكثيرة وحسب أجيال الحاسبات والمعالجات

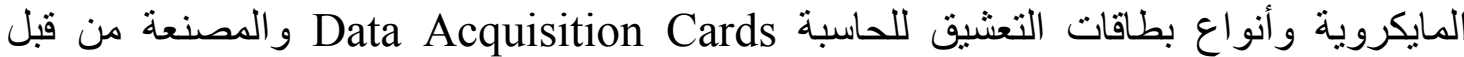

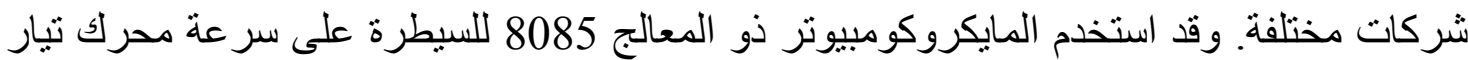

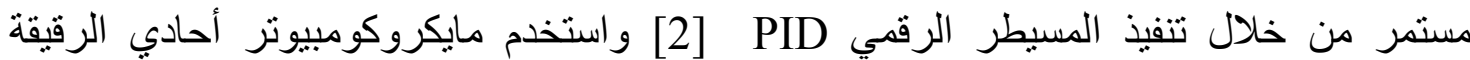

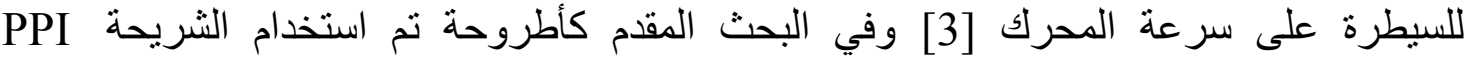
(8arallel Peripheral Interface) (8255)

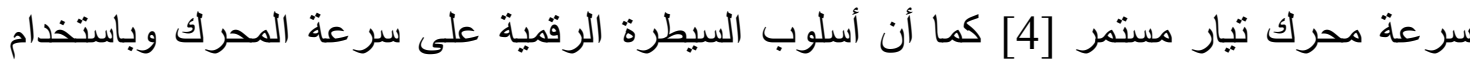

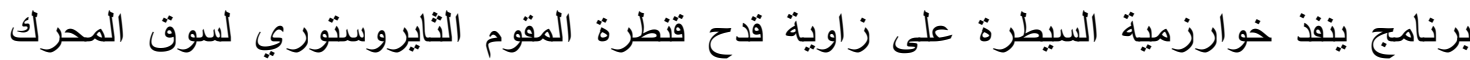

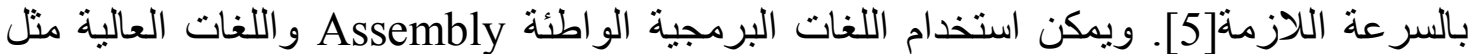

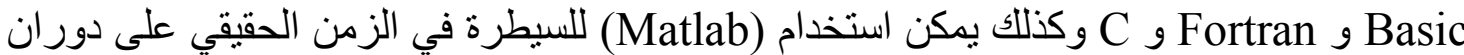

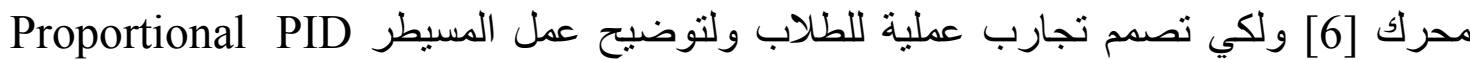
Plus Integral Derivative Controller

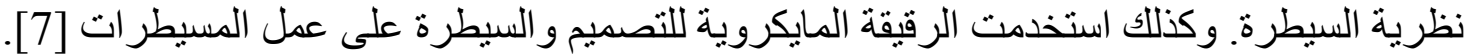

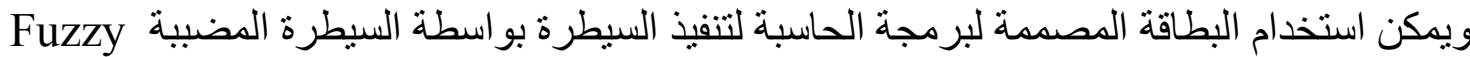
Logic Controller

تم في هذا البحث تصميم دائرة موائمة عبر الممر القياسي الصناعي ISA ( بسعة 17 بت)

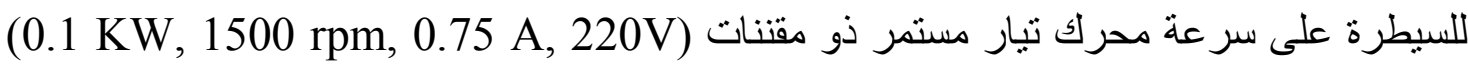

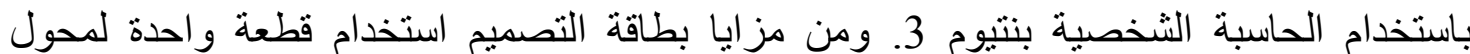
تناظري إلى رقمي AD 574 لإدخال بيانات كلا من السرعة والتيار وكتغذية خلفية عكسية إلى بلى المعالج ضمن فترة التقطيع Sample Period وتم معالجة إنشارة الخطأ باستخدام خوارزمية

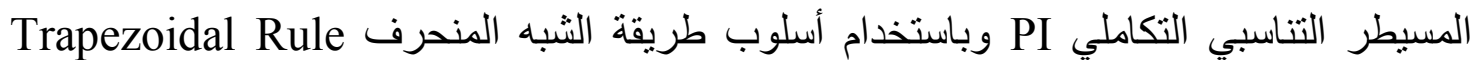
للتكامل وذات صفة استرجاعية Recursive مع تغذية عكسية للتيار لتحسين أداء المنظومة. 
يبين الثكل (1) مخططا كتلياً لمنظومة السيطرة على سرعة الدحرك. والإدخالات هي المحرك سرعة ( المقاسة بو اسطة تاكوميتر ) و التيار المار في دائرة المنتج حيث يتم تحويل هذه الإشارة إلى بيانات

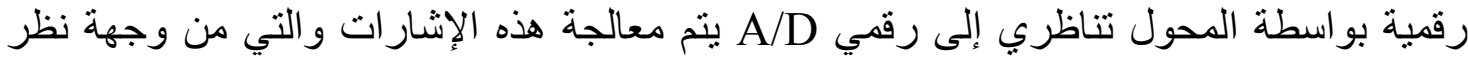

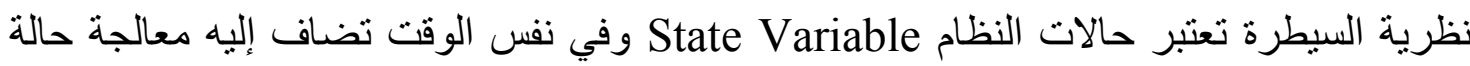

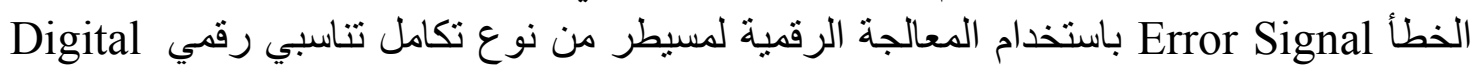

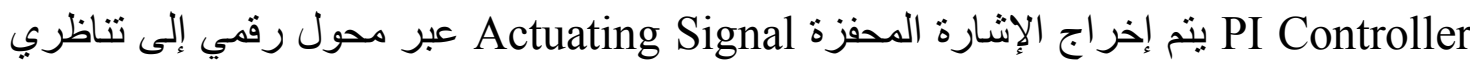

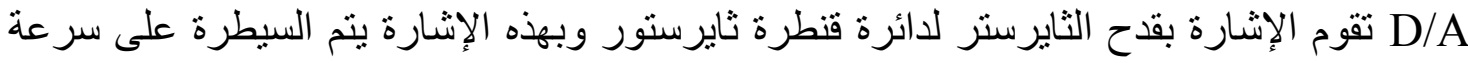

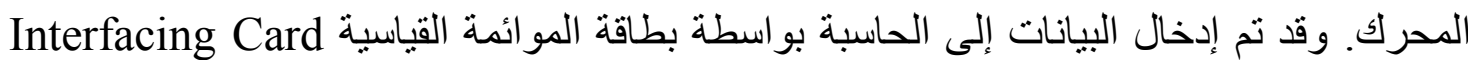

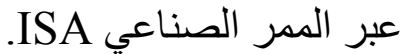

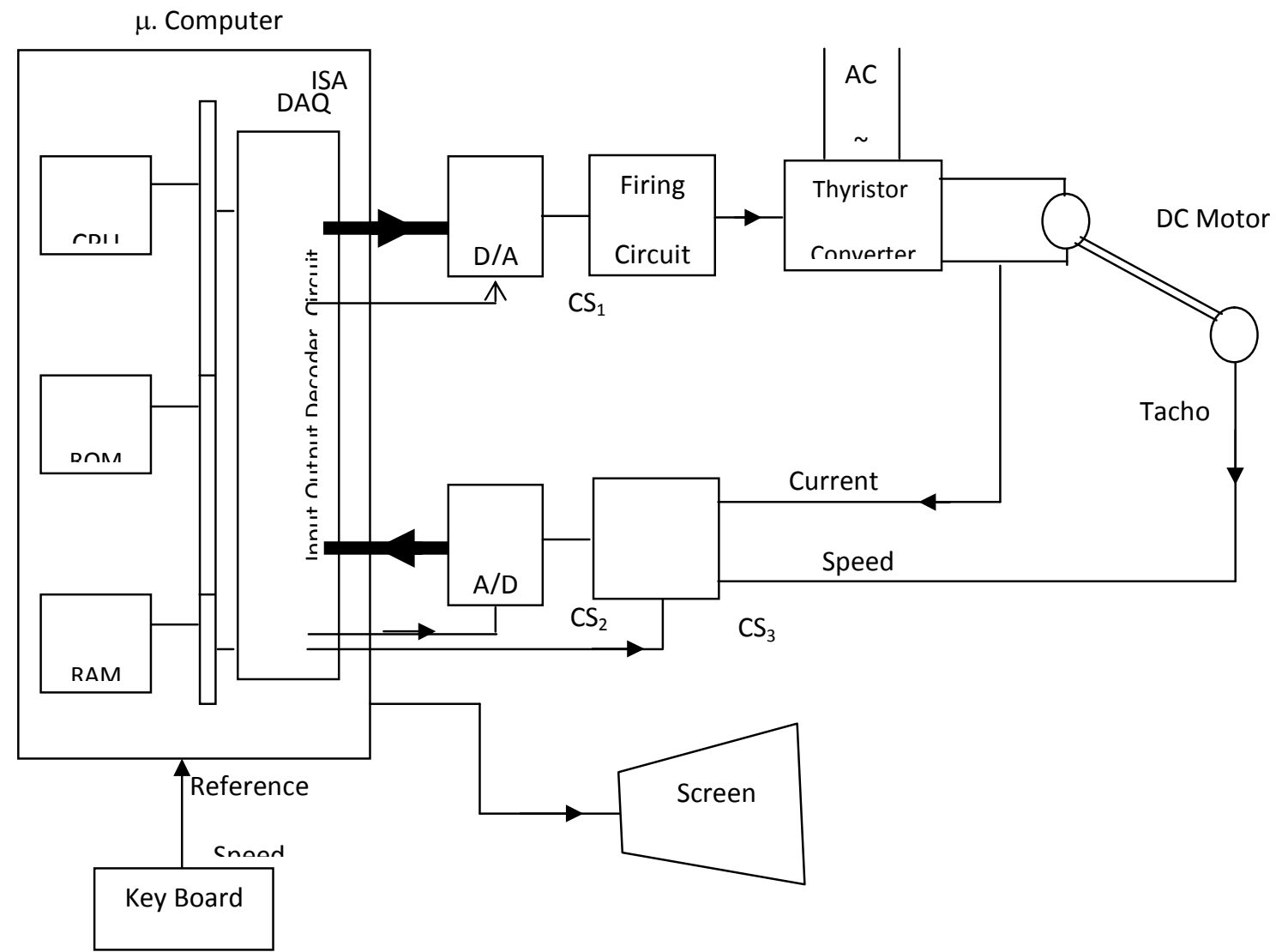


تعمل الدائرة على فلك الجفرة (Decoder) لعنوان الذاكرة Memory Address لكي يتم

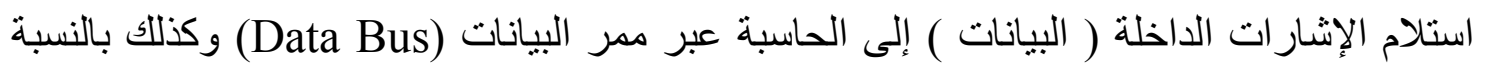

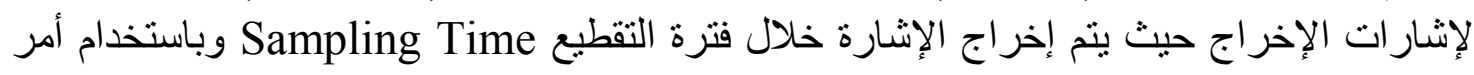

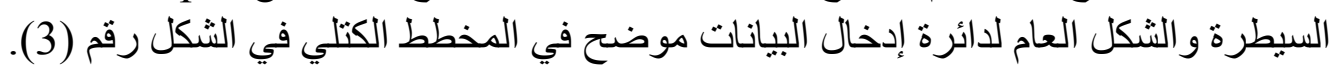


و وخند وصول العنوان الخاص إلى الخارج يتم استلام الإشارات لكل من السرعة والتيار وبالتتابع

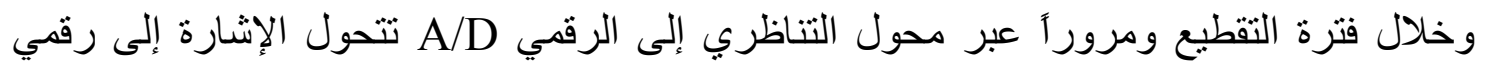

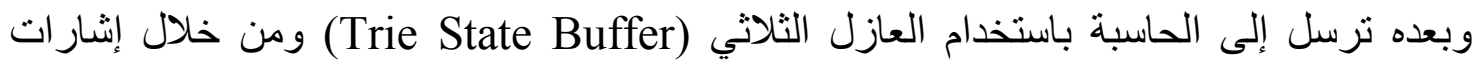
Input $\quad$ Output $\quad$ Read .Direction of Data Flow (DIR) و

وبعد إجراء المعالجة للإشار ات الداخلة لكل من التيار و السر عة يتم إخر اج الإثارة الخارجة

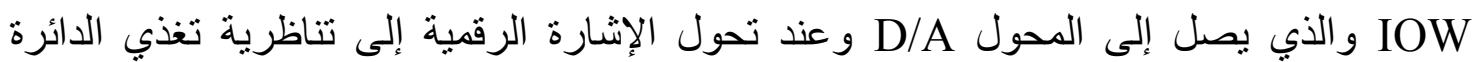

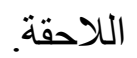


محمود : تصميم بطاقة موائمة للسيطرة على سرعة محرك تيار مستمر عبر الممر الصناعي القياسي للحاسبة

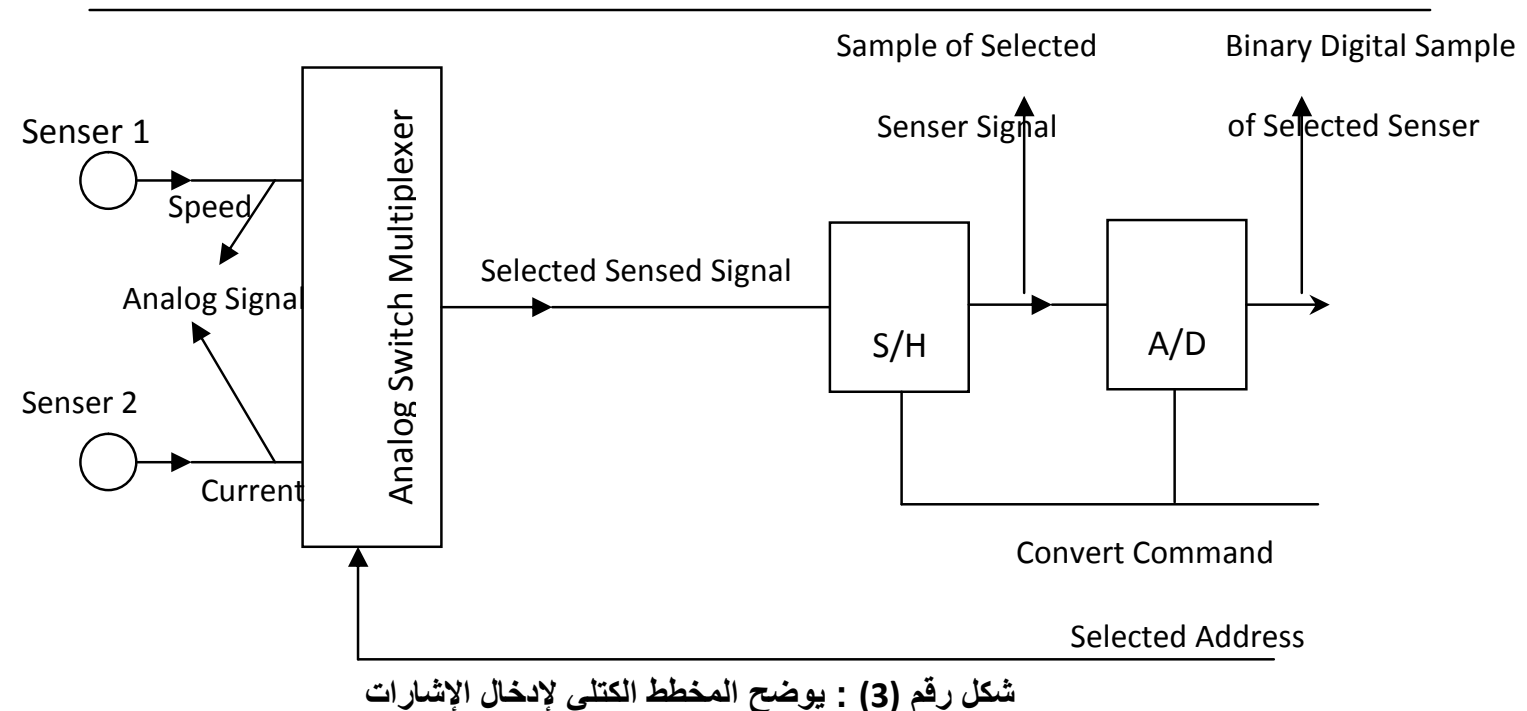

2.2 ممر البنية القياسية الصناعية ISA

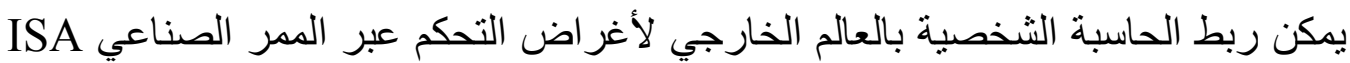

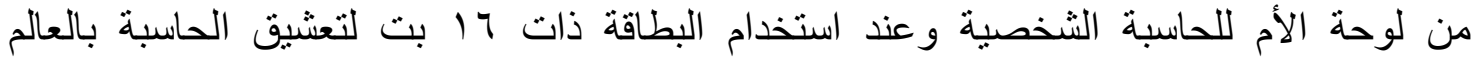

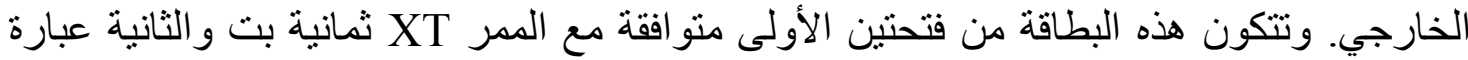

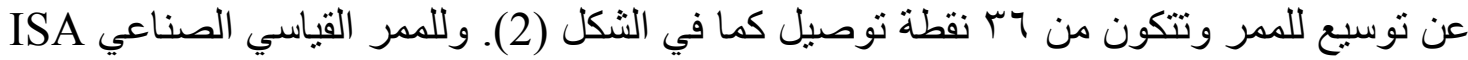

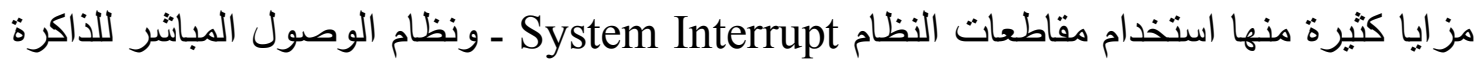
DMA لتأمين الدخاطبة و الوصول إلى المعالج الرئيسي. 


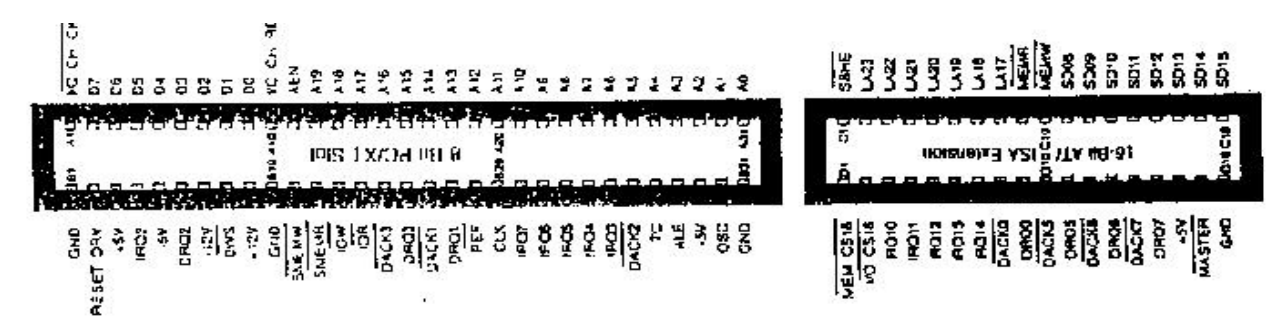

شكل رثم (2) : الفتحة التوسعية الإضافية للممر ISA

\section{3 زمن التقطي Sampling Time}

يعتبر اختيار زمن التقطيع أمراً وخطوة مهمة عند تصميم منظومة سيطرة رقمية وذلك لتلافي حدوث تداخل Aliasing وكذلك ضمان نظام مستقر. وفي منظومات السيطرة الرقمية

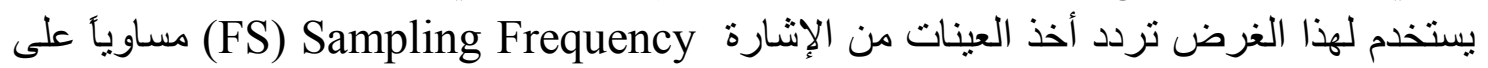
الأقل ضعف عرض حزمة الإشارة الداخلة أو يتم اتخاذ التردد (FS) مساوياً لـ عشرة أو عشئ عشرون

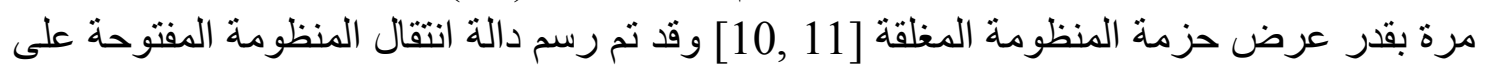
خارطة ميكلز وفيه يتم الحصول على عرض حزمة حزمة المنظومة المغلقة واختيار زمن التقطيع وكان مساوياً

2.4 مخطط لاائرة الموائمة المصممة وتركيبها :

Chip select يبين الثكل (a) مكونات عملية فلك الثفرة واختيار القطع المناسبة التالية و عددها أربع CS0, CS1, CS2, CS3 الخارجة من القطعة 74LS138. حيث بذهب CS0 بذهب إلى دائرة قلب Inverter وتوصل بعدها إلى النقطة RC في محول A/D لتهيئة البيانات إلى رقمي و إخراج الـ Status ويذهب CS1 إلى النقطتين Output enable OE في القطع 20.

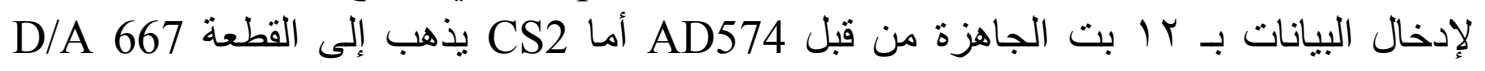
لتحويل الإشارة المسيطرة الخارجة من الحاسبة إلى تناظري Analog و

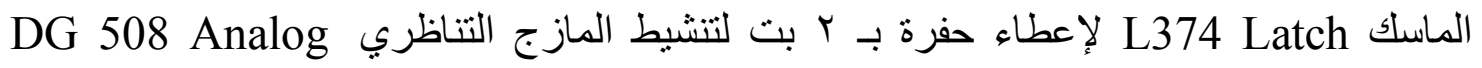

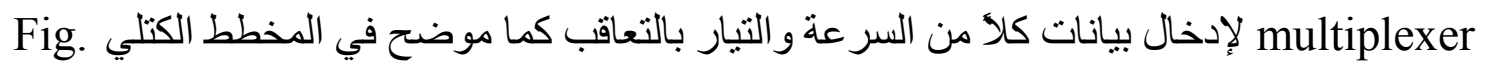


(4b). وبشكل عام مبدأ عمل الدائرة تكون كذللك ، عند نزول العنوان من الذاكرة إلى الممر

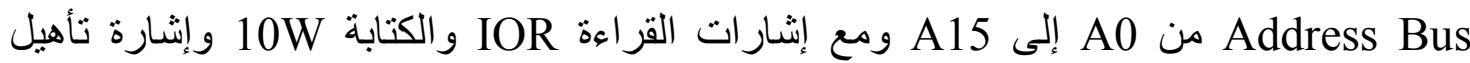
العنوان Address Enable AEN وإنشارة Aystem Bus High Enable) SBHE). ينم في

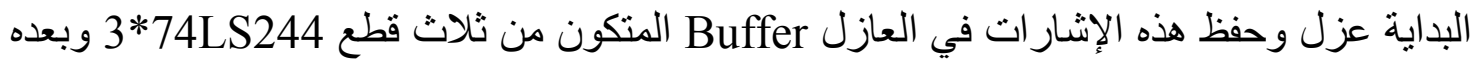

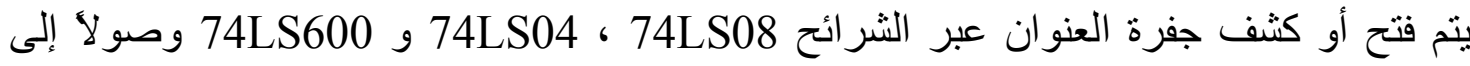

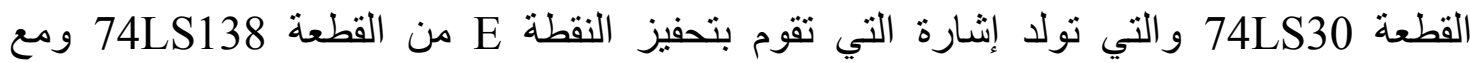

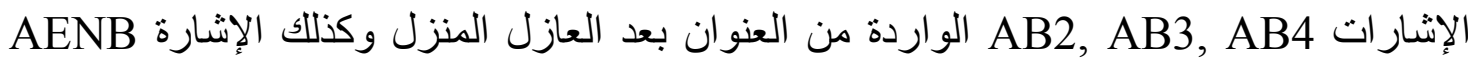

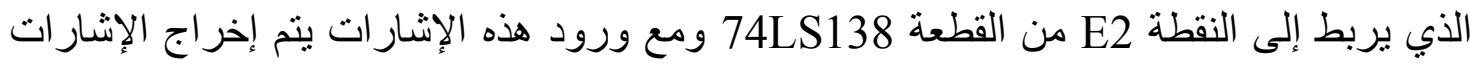

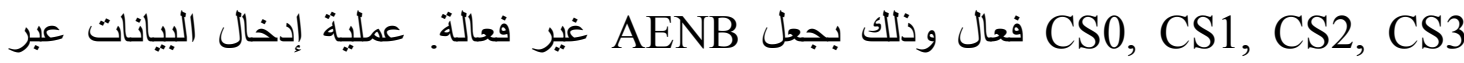

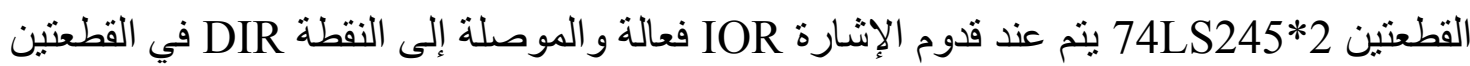

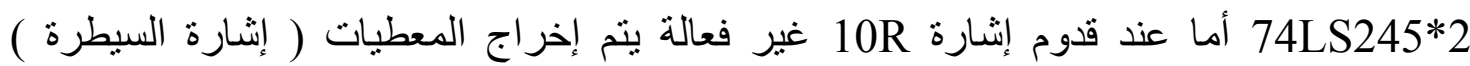
Control signal

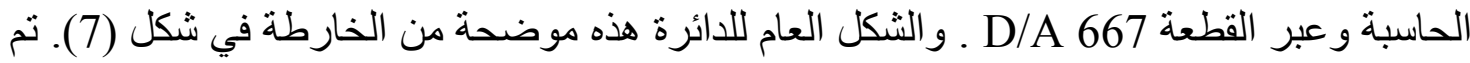

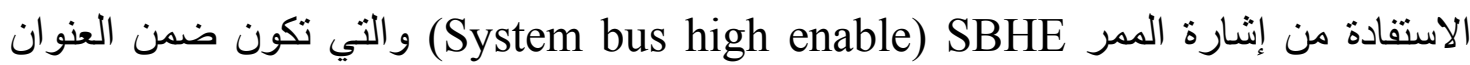

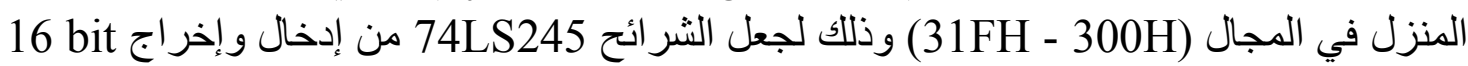

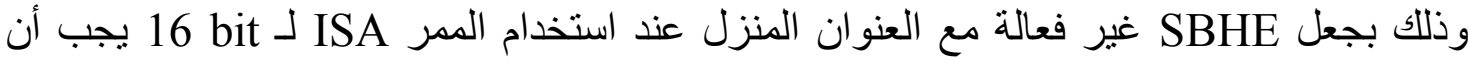

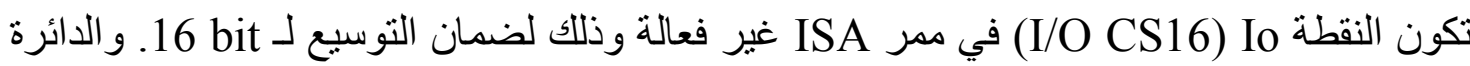

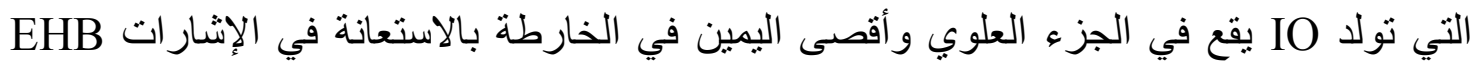

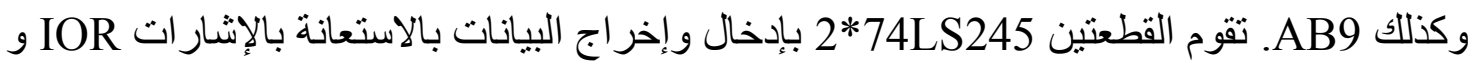

.EHB g ELB

2.5 المحول التناظري الرقمي (A/D) ومحول الرقمي إلى تناظري (D/A) :

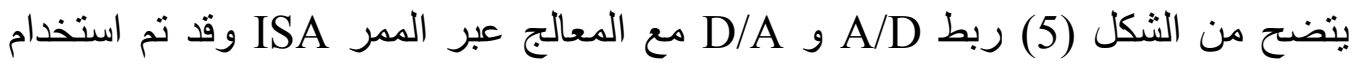

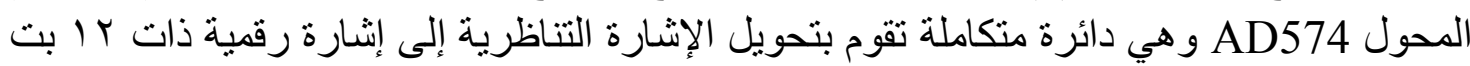

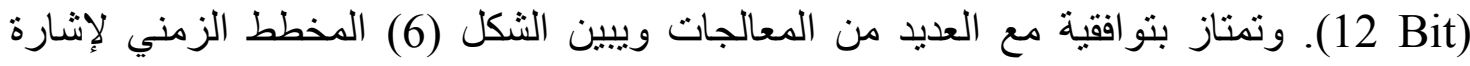

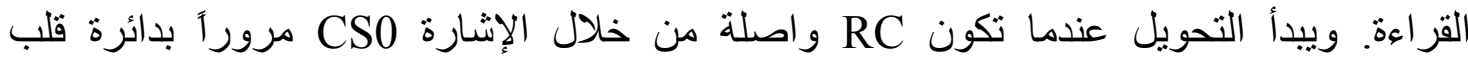

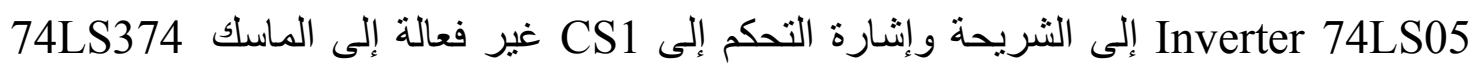

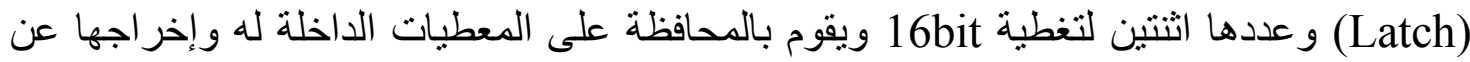

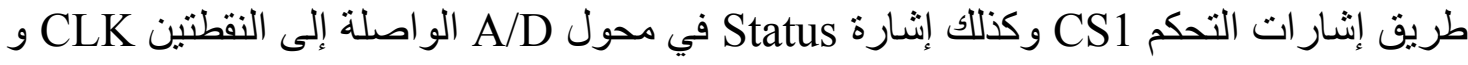
كما موضح في الرسم (4-6) 
محمود : تصميم بطاقة موائمة للسيطرة على سرعة محرك تبار مستمر عبر الممر الصناعي القياسي للحاسبة

واستخدم المحول AD667 لتحويل الإشارة الرقمية 12 بت إلى تناظرية ، تسنطيع هذه

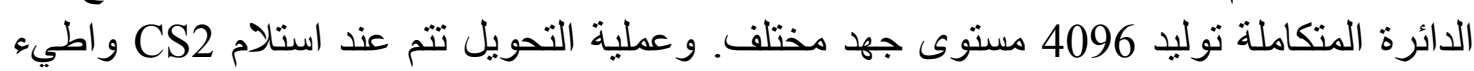
(Low) لائرة المو ائمة مكتملة. 
محمود : تصميم بطاقة موائمة للسيطرة على سرعة محرك تيار مستمرعبر الممر الصناعي القياسي للحاسبة

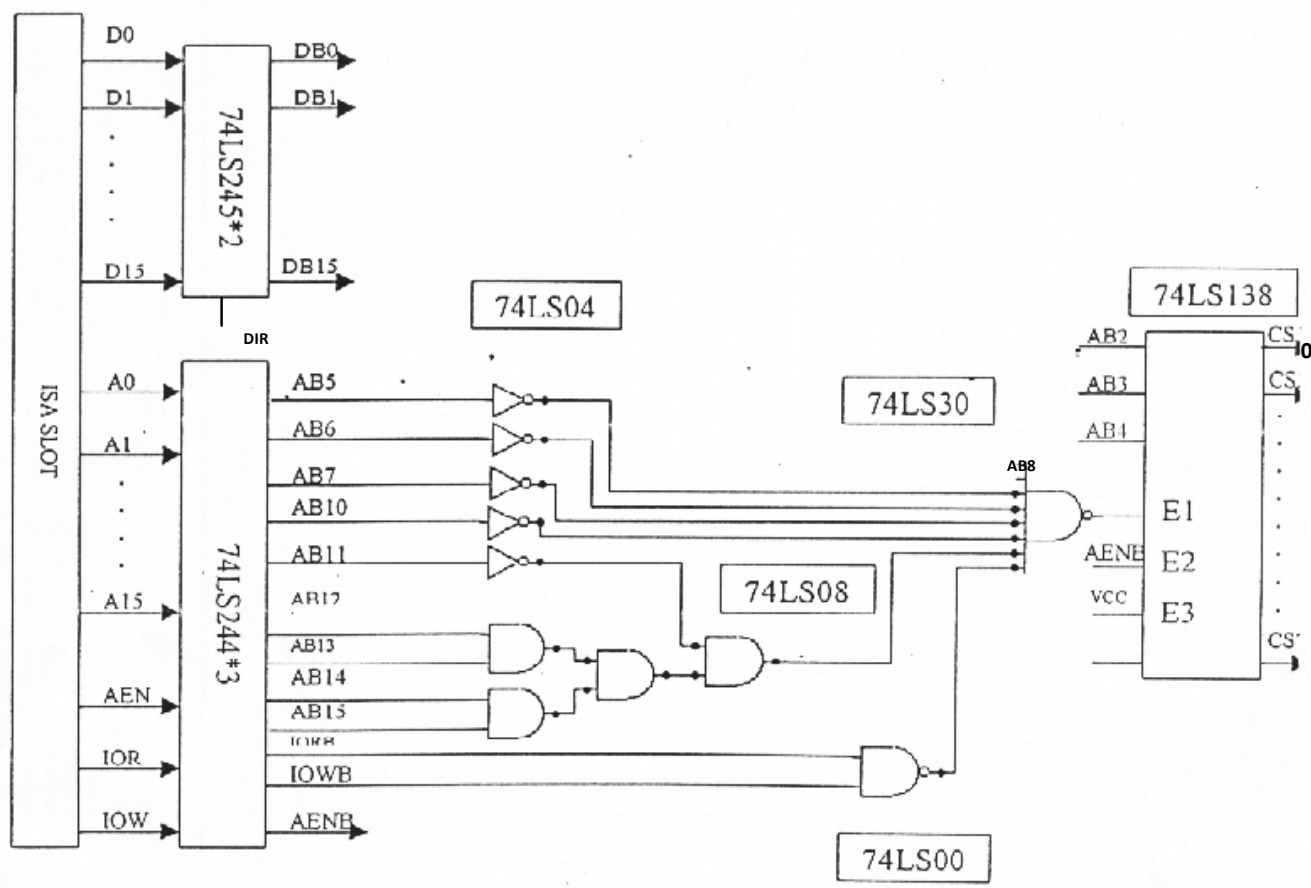

(4-a)

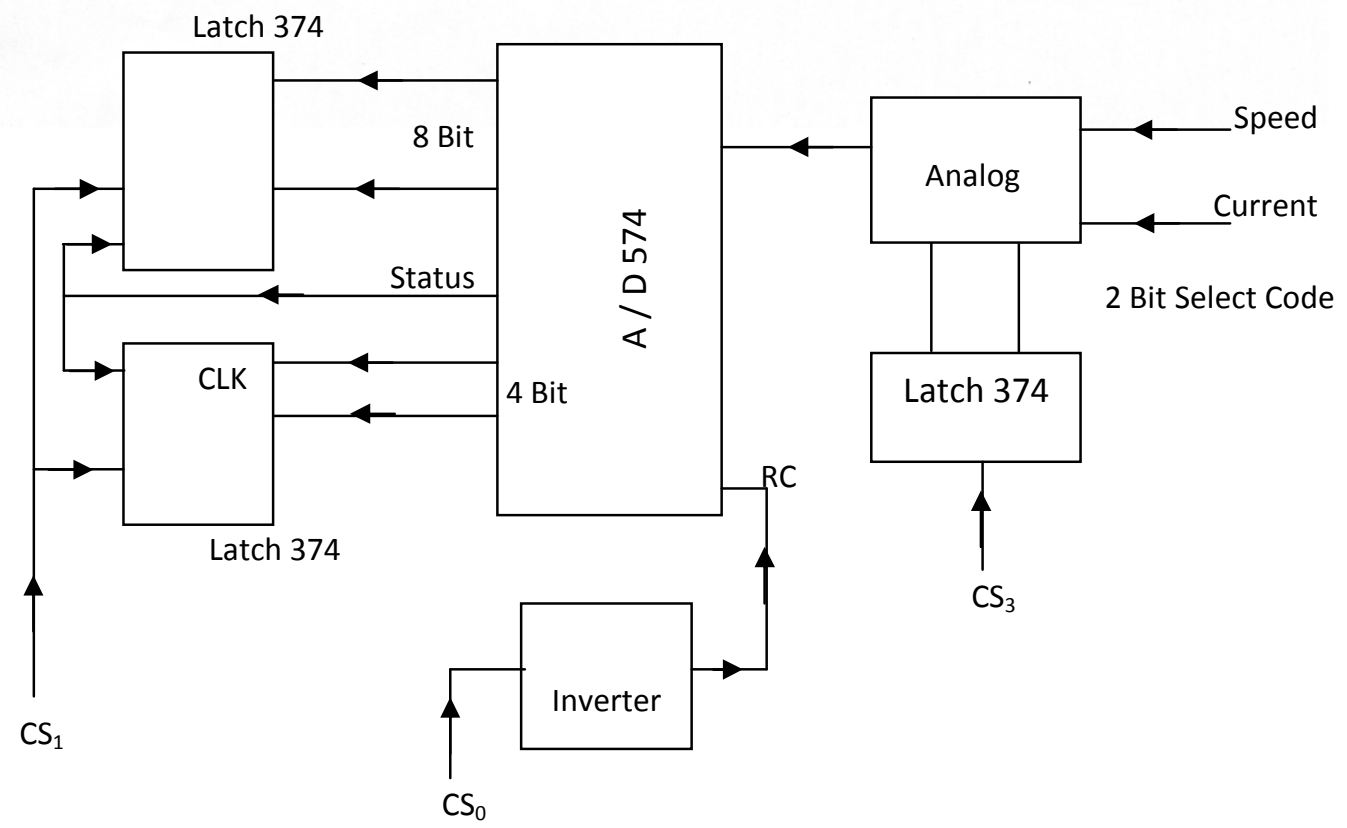

(4-b)

شكل رقم (4) : مخطط دائرة العنونة وتوليد إثـارات التحكم 
محمود : تصميم بطاقة مو ائمة للسيطرة على سرعة محرك تبار مستمر عبر الممر الصناعي القياسي للحاسبة 


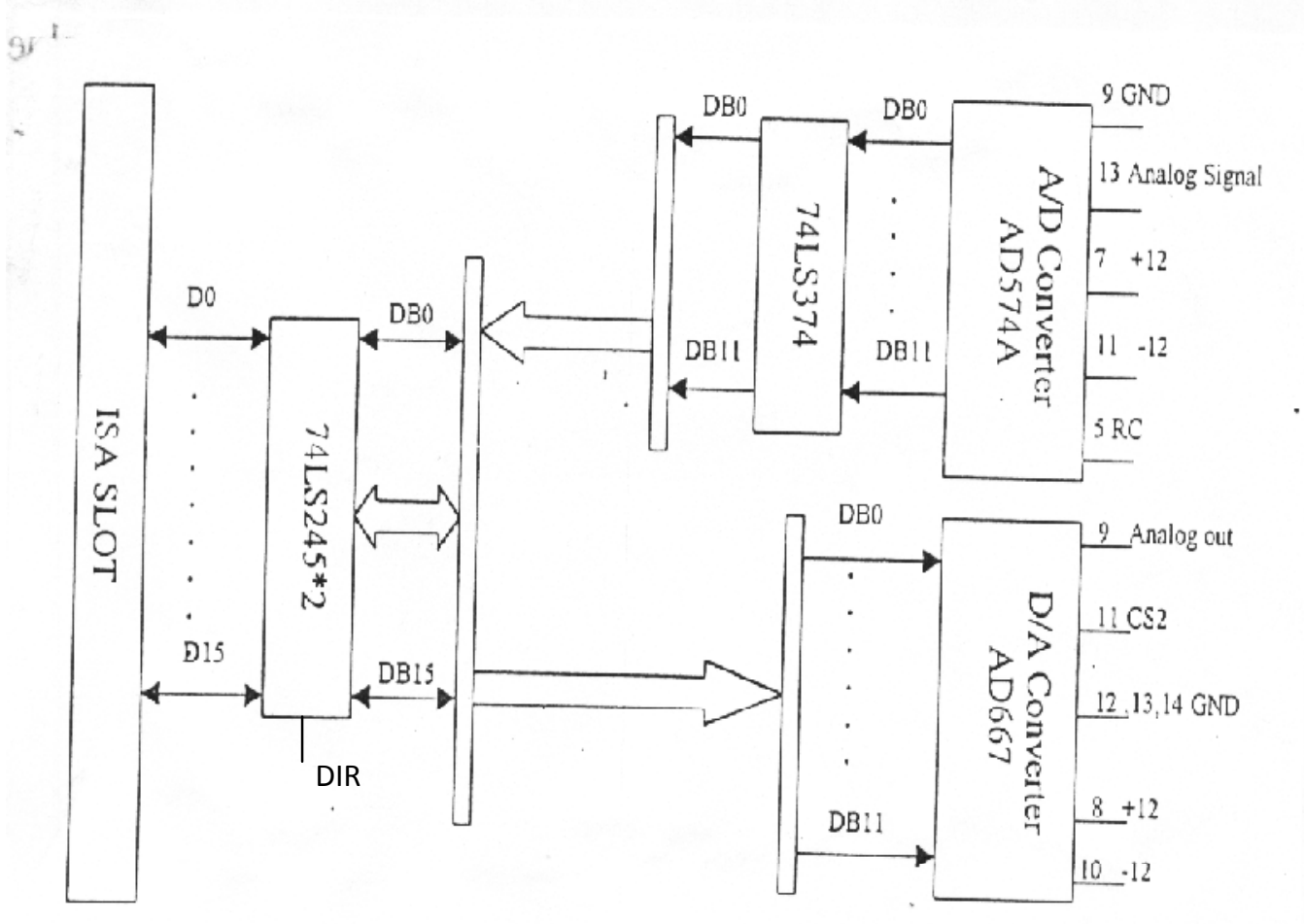

شكل رقم (5) : ربط DAC ADC مع المعالج عن طريق ISA SLOT

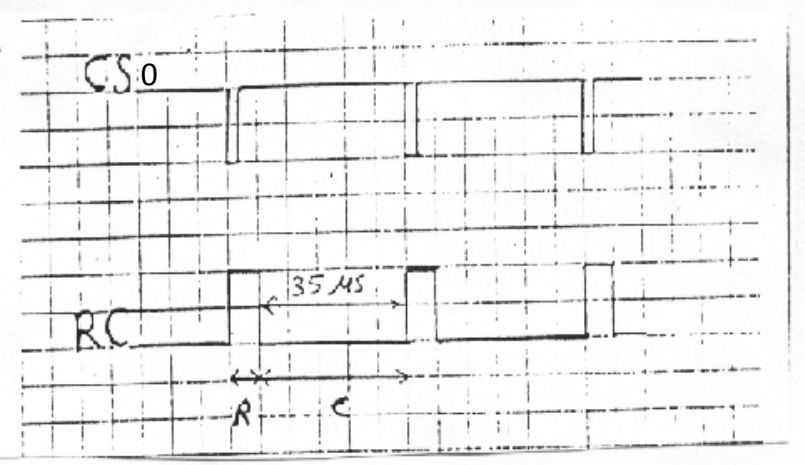

شكل رقم (6) : المخطط الزمني لإثشارة القراءة وبدء التحويل 
محمود : تصميم بطاقة مو ائمة للسيطرة على سرعة محرك تيار مستمر عبر الممر الصناعي القياسي للحاسبة

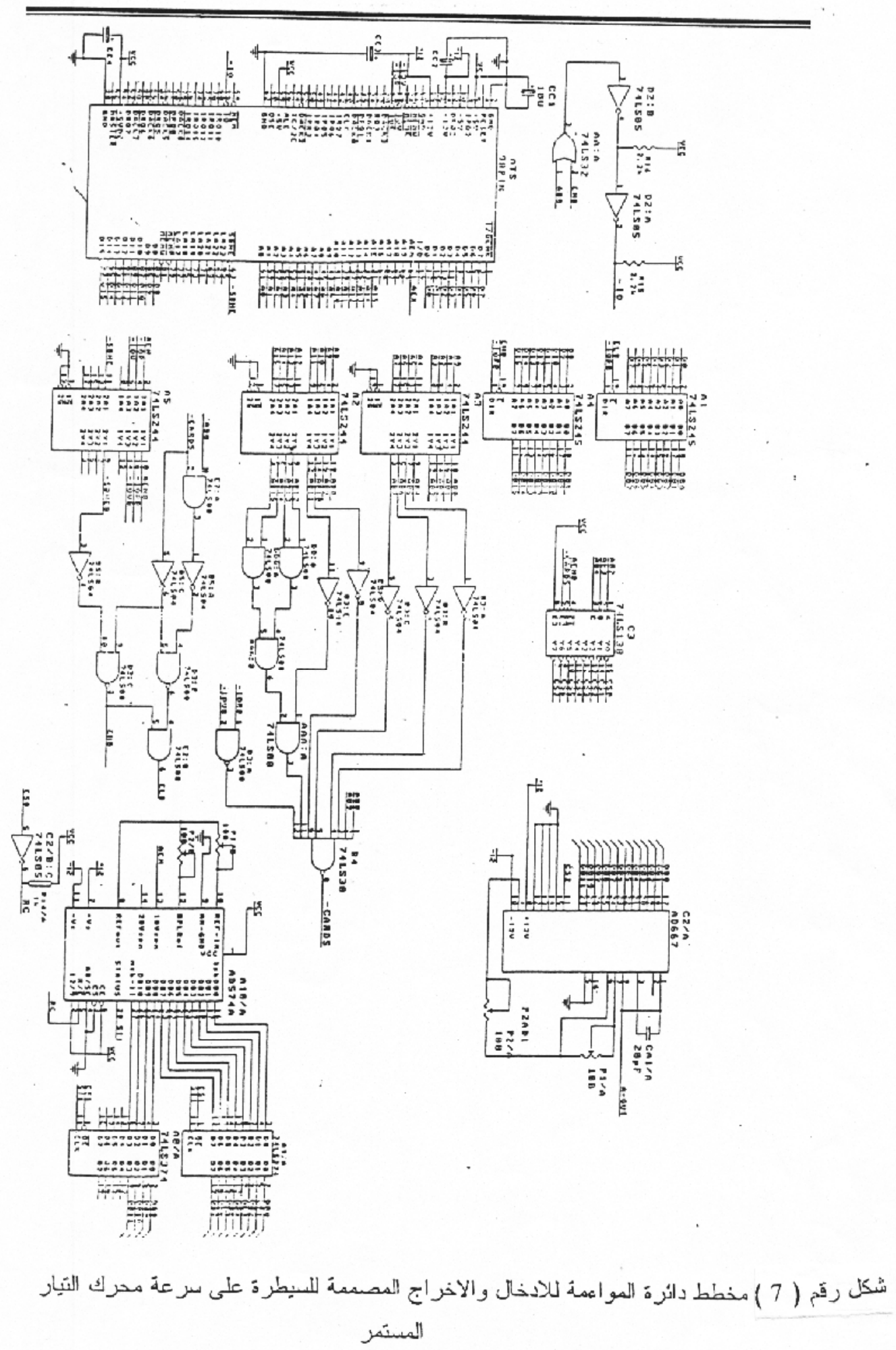


3. خوارزمية المسيطر التناسبي التكاملي والبرامجيات :

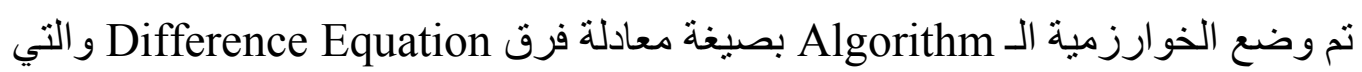
تكون مناظرة للمسيطر التناسبي التكاملي PI في المسيطرات لمنظومات السيطرة التناظرية وفي

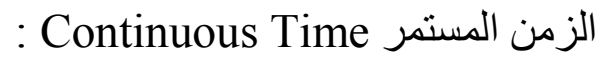

$$
\frac{M(S)}{E(S)}=K_{p}+\frac{K I}{S}=\frac{K I\left(1+S K_{p} / K I\right)}{S}
$$

حيث M(S) هو إخراج المسيطر و E(S) هو إثارة الفرق بين إثارة التغذية العكسية والإشارة المرجعية Reference Set Value Wr(s)

$$
\mathbf{E}(\mathbf{S})=\mathbf{W}_{\mathbf{r}}(\mathbf{S})-\mathbf{W}_{\mathbf{1}}(\mathbf{s})
$$

ولتحويل المعادلة رقم ( (1) إلى خوارزمية أو معادلة فرق Difference Equation لكي ينم

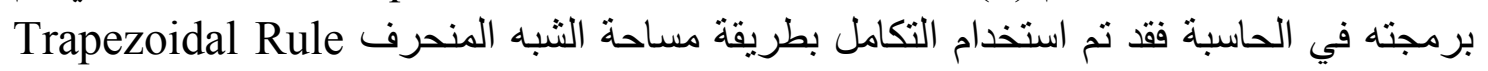

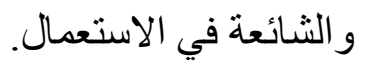

$$
\begin{gathered}
\int_{0}^{\mathrm{t}} \mathrm{e}(\mathrm{t}) \mathrm{dt} \approx \frac{\mathrm{T}}{2} \sum\{\mathrm{e}(\mathrm{kT})+\mathrm{e}(\mathrm{k}-1) \mathrm{T}\}=\mathrm{M}(\mathrm{kT}) \\
\mathrm{e}(\mathrm{kT})=\mathrm{W}_{\mathrm{r}}(\mathrm{kT})-\mathrm{W}_{1} \mathrm{kT}
\end{gathered}
$$$$
\text { علمًا أن : }
$$

Recursive وبجعل المعادلة بشكل استرجاعي Sample Period يمثل زمن التقطيع: T

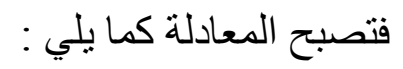




$$
\mathrm{M}(\mathrm{k})=\mathrm{M}(\mathrm{k}-1) \mathrm{T}+\mathrm{W}_{\mathrm{r}} \mathrm{T}-\frac{\mathrm{T}}{2}\left[\mathrm{~W}_{1}(\mathrm{kT})+\mathrm{W}_{1}(\mathrm{k}-1) \mathrm{T}\right]
$$

$$
\text { ومعادلة الفرق للمسيطر نوع PI يصبح كما يلي : }
$$

$$
\mathrm{U}_{1}(\mathrm{kT})=\mathrm{K}_{\mathrm{p}} \mathrm{e}(\mathrm{kT})+\mathrm{K}_{\mathrm{i}} \mathrm{M}(\mathrm{kT})
$$

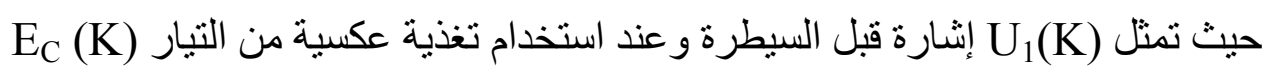

$$
\mathrm{E}_{\mathrm{C}}(\mathrm{kT})=\mathrm{I}_{\mathrm{C}}(\mathrm{kT}) \times \mathrm{R}
$$

حيث R تمثل المقاومة المربوطة على التوالي مع المنتج وتصبح إثـارة السيطرة الخارجة من الحاسبة U2 (K) عبر محول D/A ومنه إلى محفز الدائرة الإلكتروني.

$$
\mathbf{U}_{\mathbf{2}}(\mathbf{k T})=\mathbf{U}_{\mathbf{1}}(\mathbf{k T})-\mathbf{E}_{\mathbf{c}}(\mathbf{k T}) \mathbf{K I}
$$

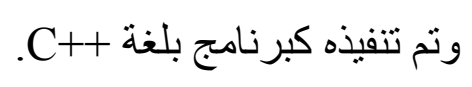

في بداية البرنامج يعطى قيم ثثابت المسيطر kp, ki, KI وكذلك السرعة المرجعية

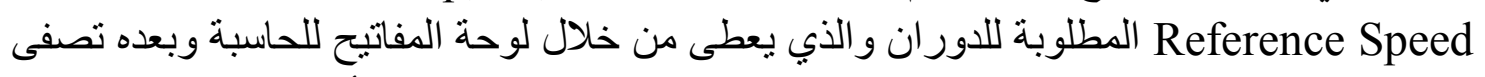

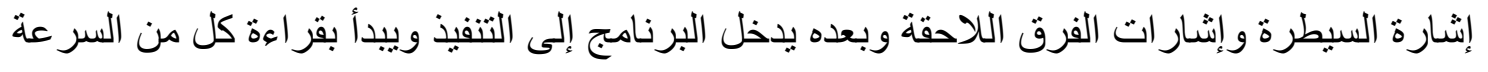

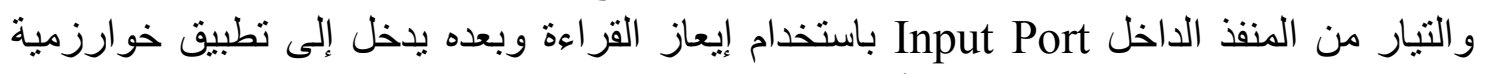

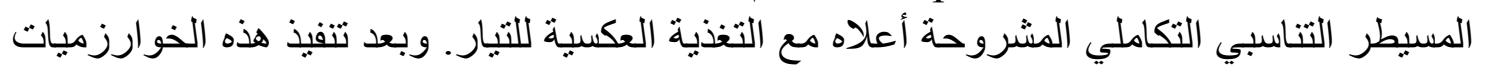

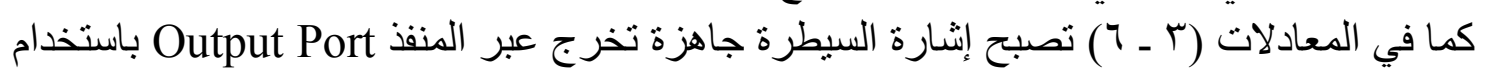
إثارة القراءة. من مز ايا هذا البرنامج يتم رسم استجابة المنظومة لكل من السرة عة والتيار على على سطح 
محمود : تصميم بطاقة مو ائمة للسيطرة على سرعة محرك تيار مستمر عبر الممر الصناعي القياسي للحاسبة

الثاشة بعد اختبار تدريجات مناسبة ولون مناسب باستخدام الإيعازات الخاصة بالرسومات

.(Graphics)

ومن إمكانيات البرنامج أيضاً بمكن تشغيل منظومة بنظام مفتوح (Open Loop) ولمدة

خمس ثو اني وبعده تغلق الدائرة ويتم رسم السرعة و التيار في الثاثة و الثكل (8) يبين خوارة ارزمية

البرنامج.

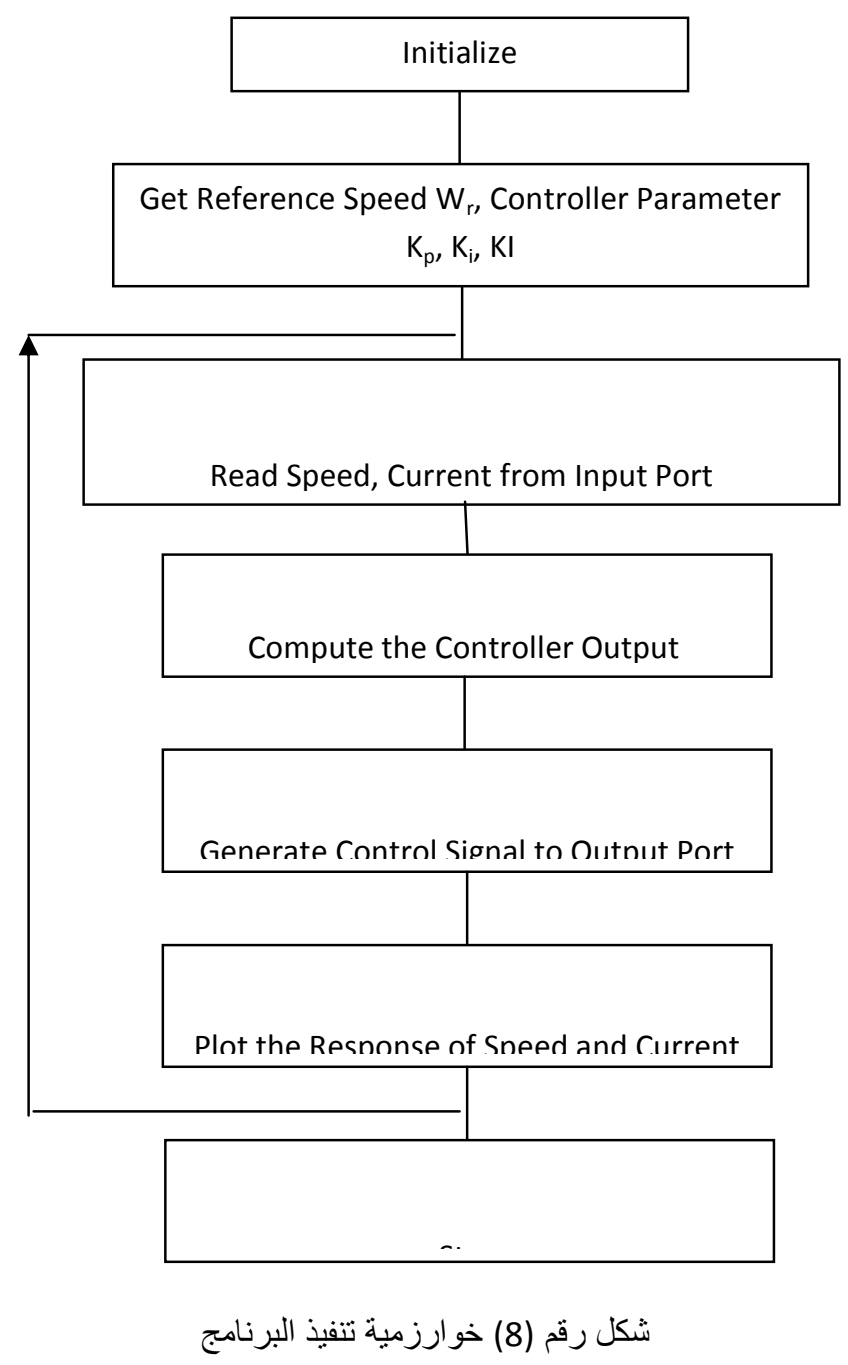




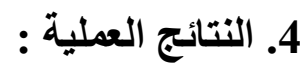

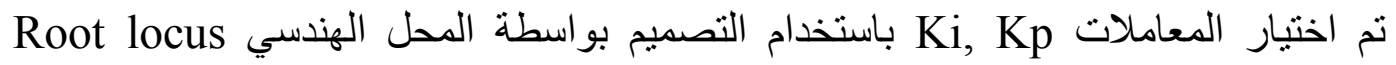

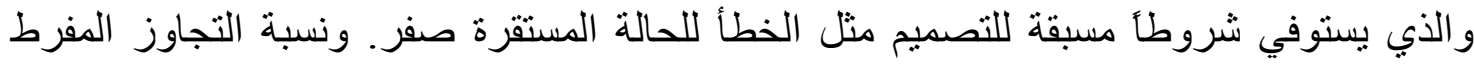
ts = اقل من 5ercent maximum overshoot kp = 2.5 وزمن الصعود أقل من . Secc.

$$
. \mathrm{KI}=0.5,10, \mathrm{ki}=2
$$

لقد تم تشغيل منظومة المسيطر على سرعة محرك التيار المستمر ولسرع مختلفة ولقيم

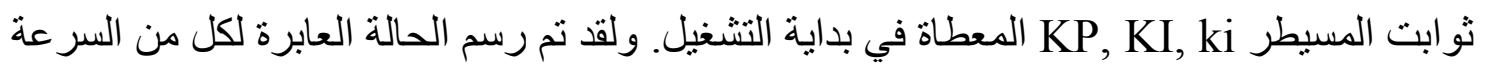

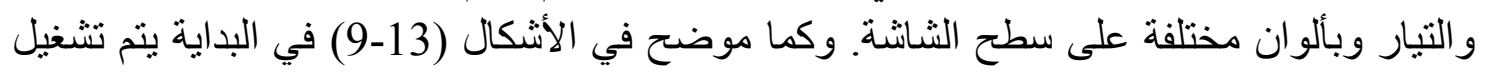

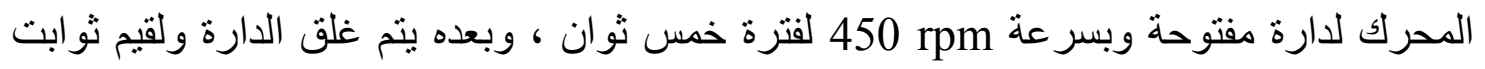
المسيطر المعطاة في بداية البرنامج ولسرعة لدارة 750 rpm المطلوبة.

لقد تم استخدام الدائرة الموائمة المصممة للسبطرة على سرعة محرك الته التيار المستمر وتم

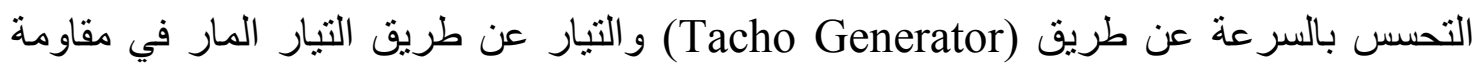

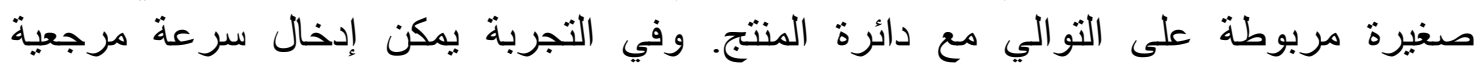
Reference Set Value

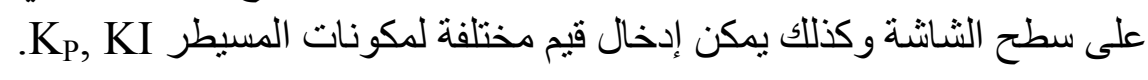

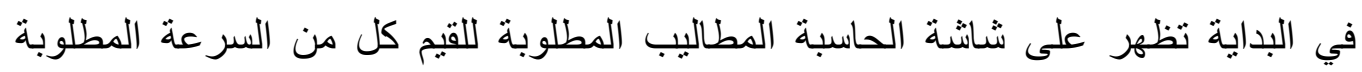

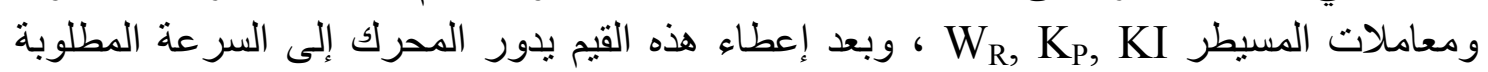

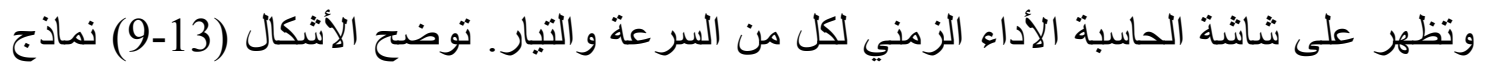

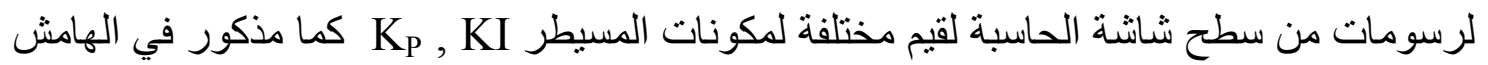
وكذللك عند تحميل المحرك.

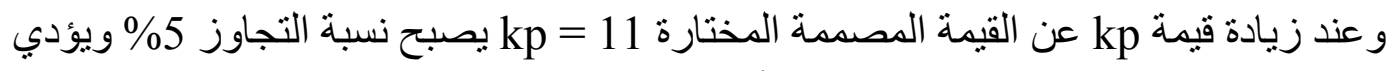

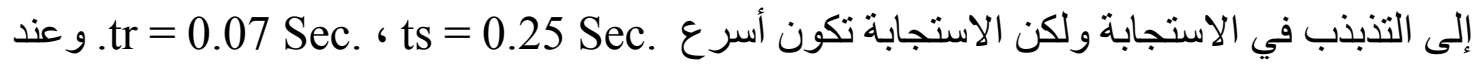

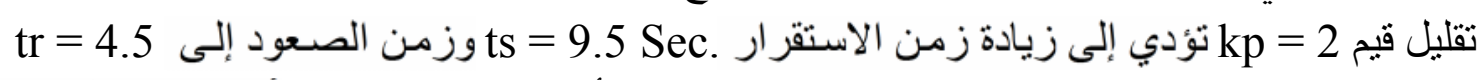

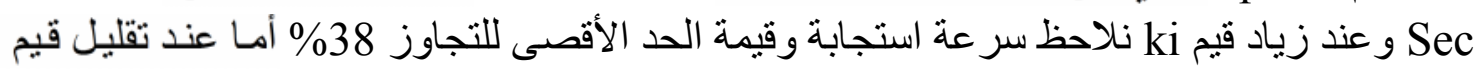

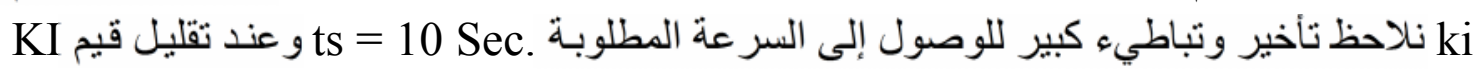

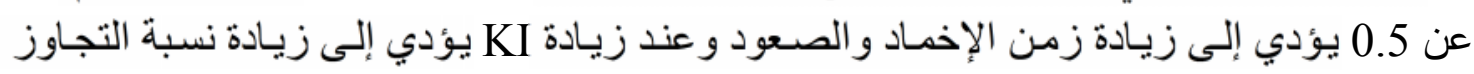
وظهور التذبذب ولكن الاستجابة تكون أسر ع. لأنع. 


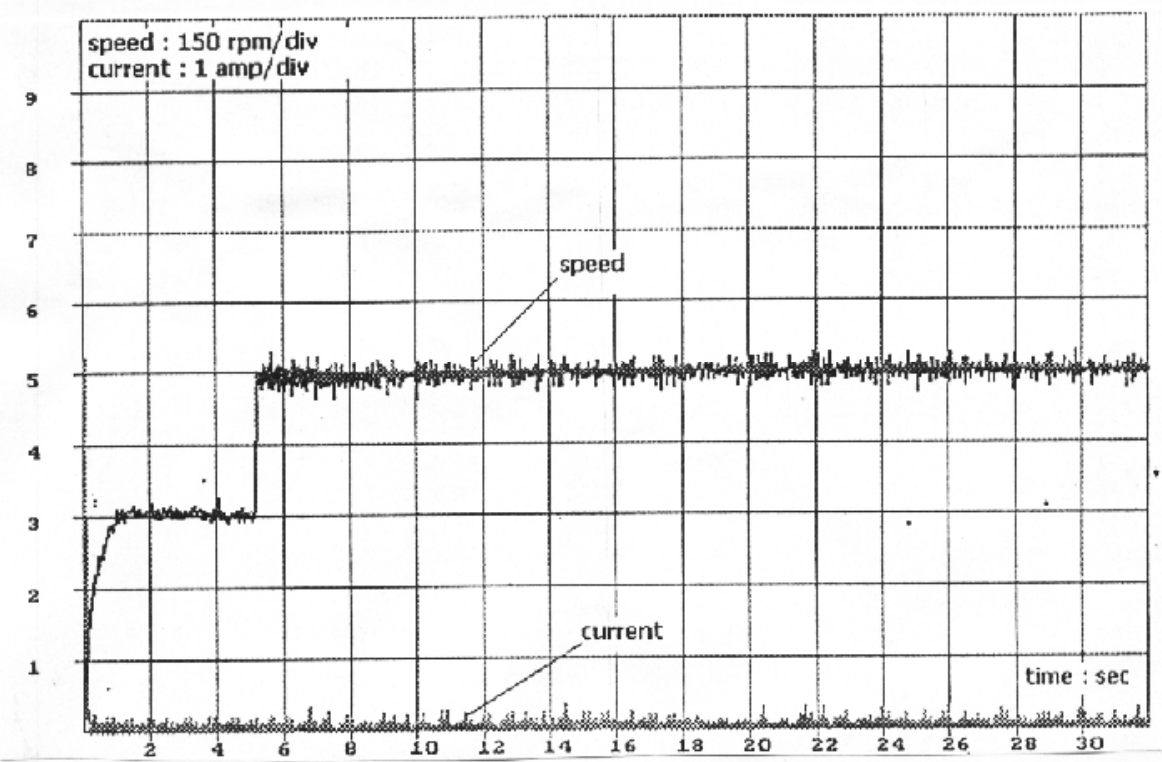

Spced, Current, Responsc: Proportional Control Kp $=10$, Integral Control $\mathrm{Ki}=2$, Current Control $\mathrm{KI}=0.5$

شكل رقتم (9) : استجابة النظظام الفكونات السبطرد المخثارة.

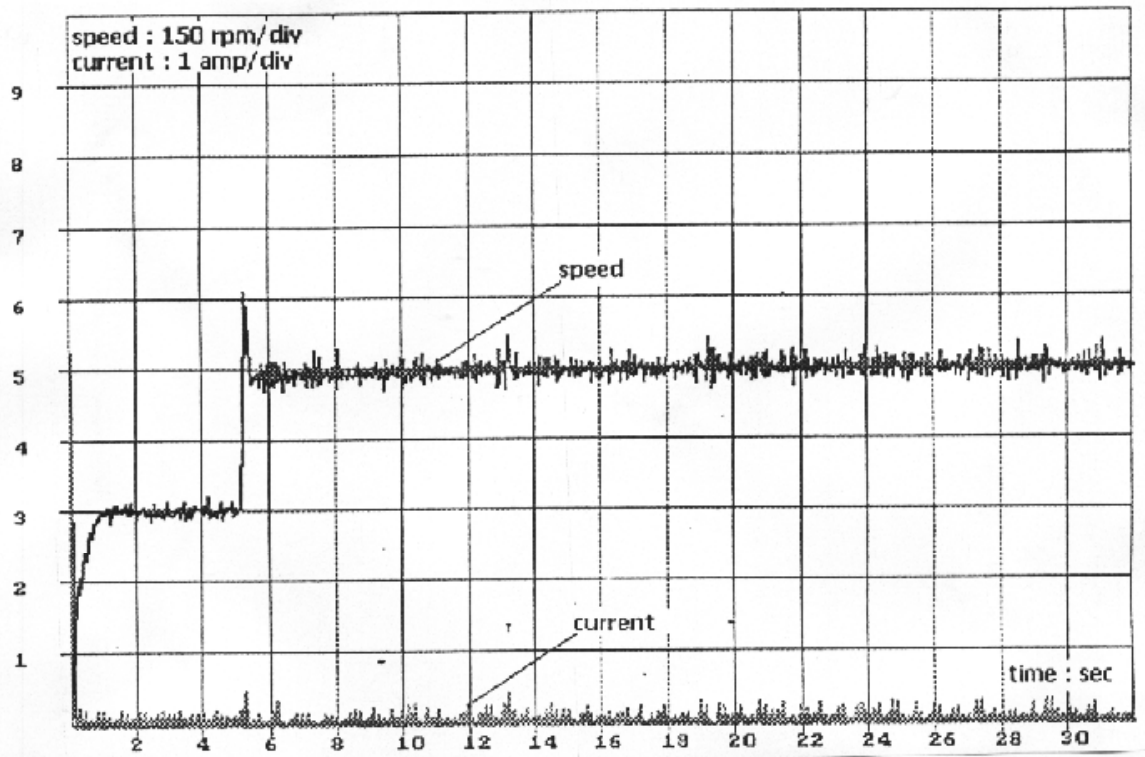

Speed, Current, Response: Proportional Control Kp $=11$,

Integral Control $\mathrm{Ki}=2$, Current Control $\mathrm{KI}=0.5$.

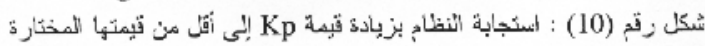




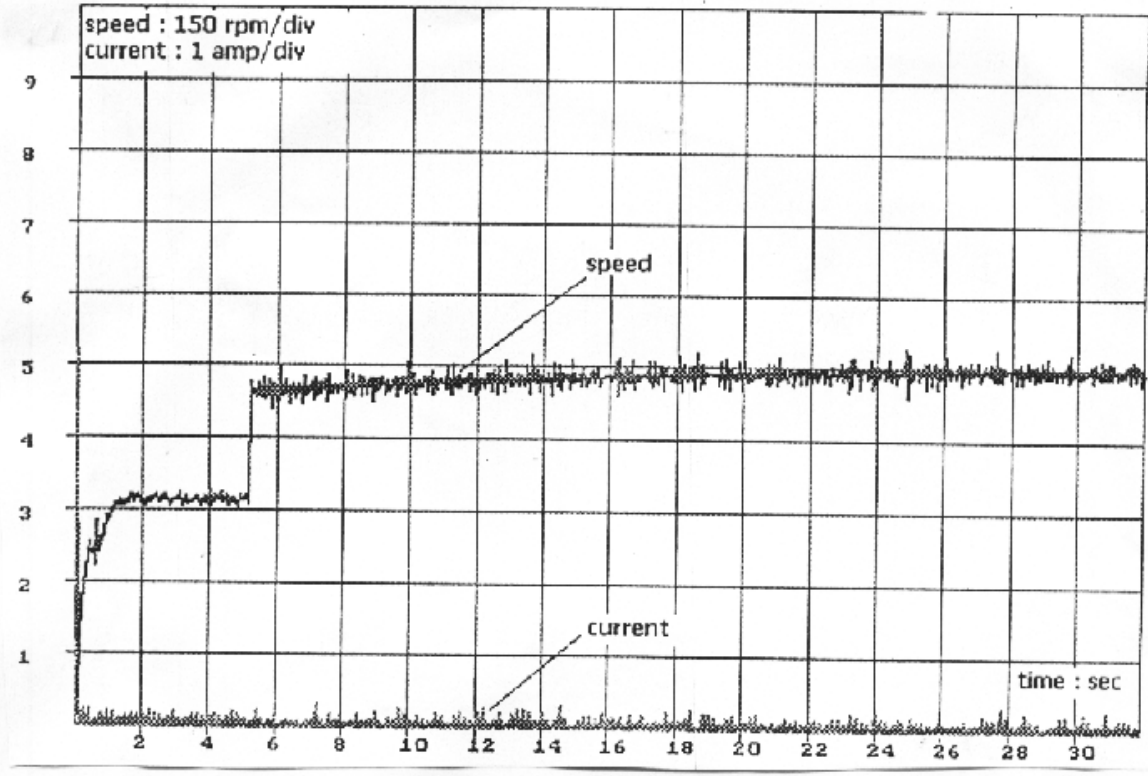

Speed. Current. Response: Proportional Control $\mathrm{Kp}=10$. Integral Control $\mathrm{Ki}=1$, Current Control $\mathrm{KI}=0.5$.

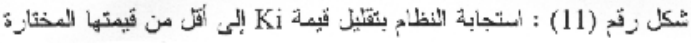

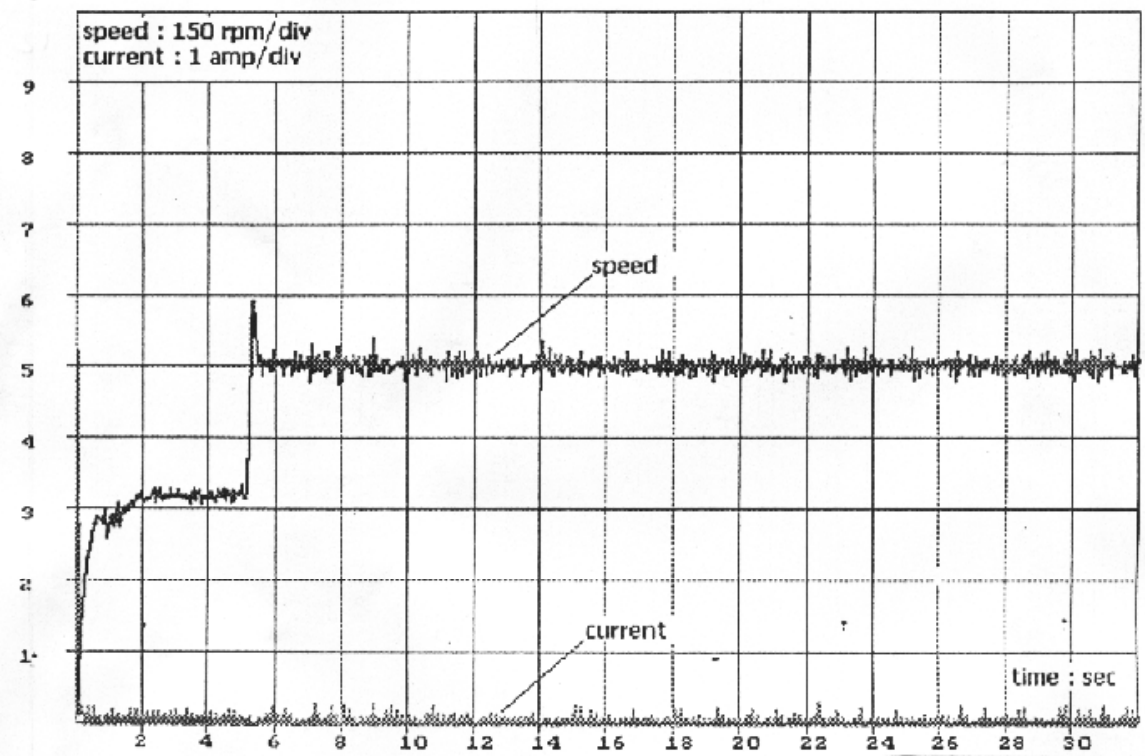

Speed, Current, Response: Proportional Control Kp $=10$

Integral Control $\mathrm{Ki}=2.499$, Current Control $\mathrm{KJ}=0.1$.

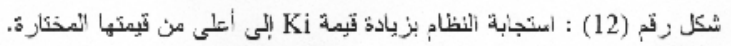




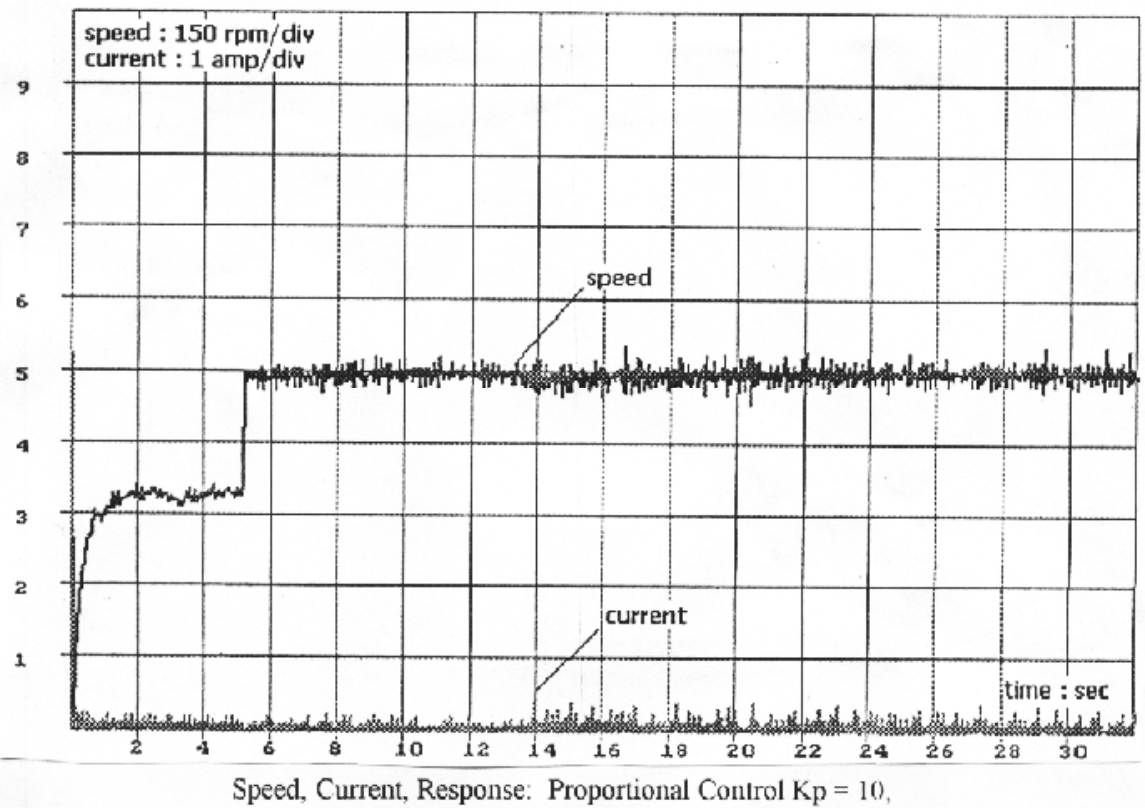
Integral Control $\mathrm{Ki}=2$, Current Control $\mathrm{KI}=0.5$.

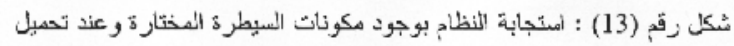

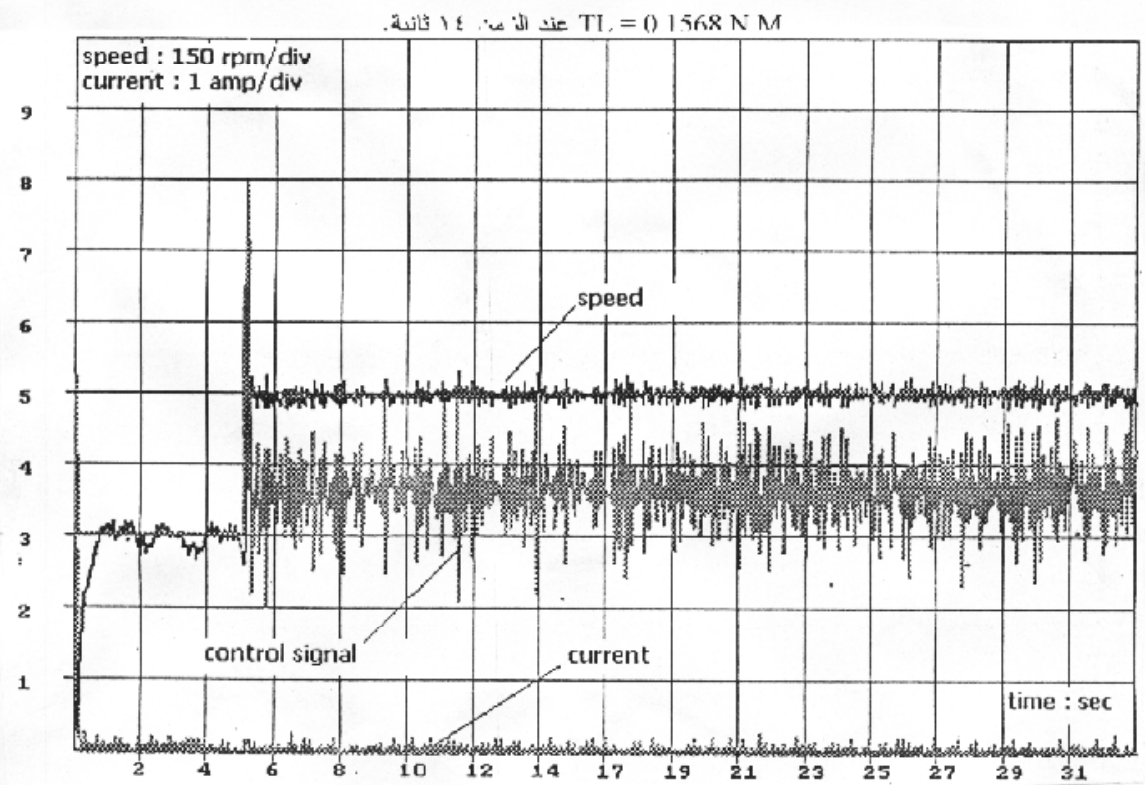

Speed, Current, Response: Proportional Control $\mathrm{Kp}=10$, Integral Control $\mathrm{Ki}=2$, Current Control $\mathrm{KI}=0.2$.

نبكل رتجّ (14) : استجابة النظاج لمكونات العبطرة المختارة بوجود إثارة العبطرة 
1. ان استخدام الحاسوب أو المسيطر الدقيق Microcontroller) يكون أكثر دقة

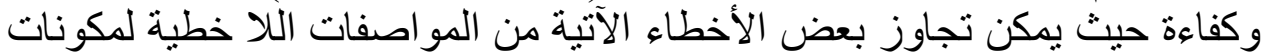

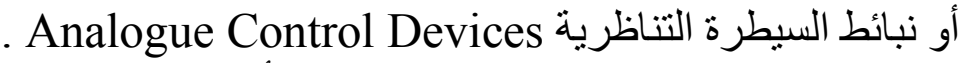
r. إن استخدام الحاسوب في السيطرة ذو مرونة أكثر بالمقارنة مع المنظومة التناظرية حيث يمكن تغيير قيم مكونات المسيطر في البرنامج المنفذ لخوارزمية المسيطر وبسهولة حبي ولمر اتب تئبر

عديدة بعد الفارزة. ومن خلال برنامج الرسومات Graphics يمكن مشاهدة

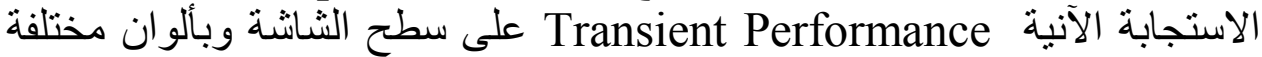

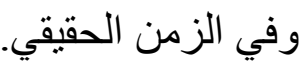

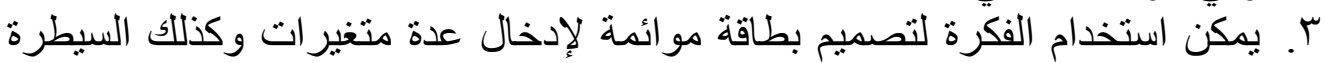

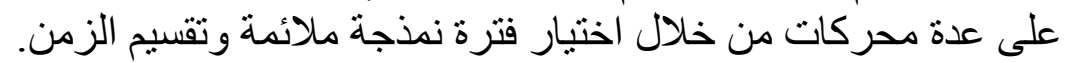

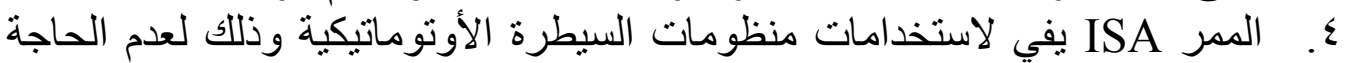

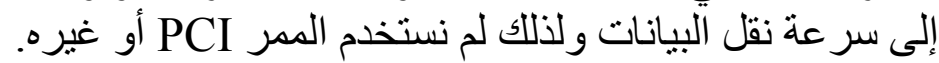

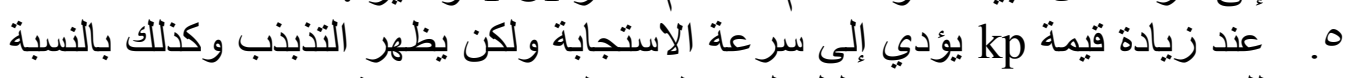
اللمعاملات ki ، KI و عند تقليل القيم يظهر بطء في الاستجابة.

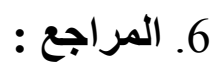

1. لويس سي اجبربخت " ربط الحاسبات الثخصية بالعالم الخارجي لأغر اض التحكم و القياس " ترجمة المهندس حيان السيد ، 1997 ، دار النشر ـ سوريا.

2. H. D. Maheshappa, R. D. Sudhaker Samuel and A. Prakashan, "Digital PID controller for speed control of D.C motors", IETE technical review, Vol. 6, No. 3, 1989, PP. 171-175.

3. J. F. Abubry, G. H. J. P. Louis \& A. A. El-hefinawy, "Minimal Microprocessor Hardware for direct digital control of A. D.C motor", control and computer, Vol. 9, No. 3, 1981, PP. 41-44.

4. Basill Asaad Matti, "Speed Control of D. C. Motor with High Accuracy Using Microcomputer", Thesis submitted to the Electrical Engineering Department, University of Technology, 1989. 
5. B. A. White \& A. R. Daniels, "Digital control of D.C motor", North-Holland System and control series, Vol. 7, 1985, PP. 253-272.

6. Yao-Chon chen and Jason M. Naughton, "An Undergraduate Laboratory platform for control system design, simulation and implementation", IEEE control system, Vol. 20, No. 3, June 2000, PP. 12-20.

7. John B. Peatman, "Design with Microcontroller". McGrawHill, 1989.

8. N, Govind and A. R. Hasan, "Real Time Fuzzy Logic Speed Control Using Conventional, Assembly and Simulation Methods for Industrial DC Motors" In Proceeding of the 1995 IEEE/IAS international conference on industrial Automatic and control, IA \& C 95, Jan. 1995, pp. 203-208.

9. T. NASSER, M. TAALABI ... etc. "Design and application of Fuzzy logic for the Speed Control of Direct-Drive DC Motor", $4^{\text {th }}$ int. Conf. CATTAEE-2002, March, 19-21, 2002, Amman, Jordan.

10.A. Frank D. Souza, "Design of control system", Prentice-Hall, INC, Englewood cliffs, New Jersey, 1988.

11.Dorf. R. C., Bishop, R. H. "Modern Control System", $9^{\text {th }}$ ed. Prentice-Hall Inc., 2001. 
محمود : تصميم بطاقة مو ائمة للسيطرة على سرعة محرك تيار مستمر عبر الممر الصناعي القياسي للحاسبة 